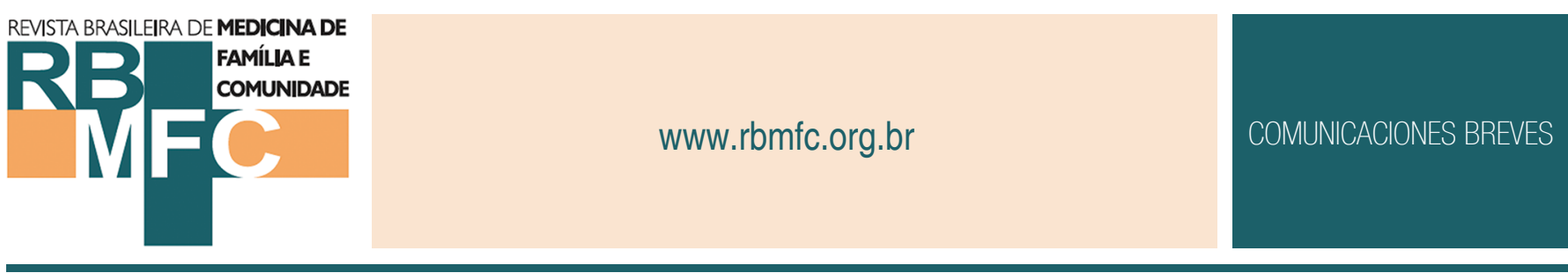

\title{
Carta de Cali, Colombia 2018
}

Durante los días 13 y 14 marzo de 2018 en Cal, Colombia se realizó la VII CUMBRE IBEROAMERICANA DE MEDICINA FAMILIAR con el tema: "CUARENTA AÑOS DE ALMAATA: MEDICINA FAMILIAR Y SALUD DE LA FAMILIA, UN CAMINO PARA LA PAZ".

Este evento, organizado por el Ministerio de Salud Pública de Colombia, la Organización Mundial de Médicos de Familia (WONCA), Confederación Iberoamericana de Medicina Familiar (CIMF), la Sociedad Colombiana de Medicina Familiar (SOCMEF, el Municipio de Santiago de Cali, y la Organización Panamericana de la Salud/Organización Mundial de la Salud (OPS/OMS) tuvo como objetivo contribuir al fortalecimiento de las políticas de salud en el campo de la Atención Primaria y la Medicina Familiar en Iberoamérica. Representantes de los Ministerios de Salud de Iberoamérica representantes de WONCA, representantes de WONCA Iberoamérica-CIMF, representantes de la SOCMEF, representantes de salud de Gobiernos Municipales de Colombia y de otros países iberoamericanos, coordinadores/as de programas docentes de Medicina Familiar, delegados/as de la sociedad civil y representantes de la OPS/OMS, analizaron los temas detallados a continuación:

1. Cuarenta Años De Alma Ata: Medicina Familiar y Salud Familiar, un Camino Para La Paz

2. Investigación aplicada a los Territorios

3. Impacto Económico de la Medicina Familiar en los Sistemas de Salud

4. Medicina Familiar y Comunitaria como fuente de Atención en Salud Mental

5. Prevención Cuaternaria: cómo hacer y cómo enseñar

6. La Medicina Familiar y Comunitaria y la Salud de los Migrantes

Producto de este análisis de los grupos de trabajo conformados por especialistas de las áreas mencionadas de las organizaciones convocantes de las instituciones que avalan esta Cumbre, se generaron las siguientes definiciones y recomendaciones:

1. Incluir obligatoriamente en los programas de pregrado y post-grado en Medicina Familiar contenidos teórico- prácticos necesarios y adecuados a cada nivel de formación, para el desarrollo de las competencias (conocimientos, destrezas, habilidades y actitudes), incluyendo la comunicación y el abordaje familiar y comunitario, para manejar la diversidad de violencias en el contexto de la práctica profesional.

2. Instar a las autoridades, en los espacios de formación y de práctica profesional, a promover el autocuidado y la paz interior para las familias y comunidades para la construcción e implementación de políticas públicas y educativas transversales al desarrollo humano, enfocado en la cultura de la paz, desde una perspectiva de derechos humanos en un trabajo intersectorial y transdisciplinario. 
3. Establecer políticas de financiamiento que fomenten la investigación para identificar los factores protectores y deteriorantes vinculados a la violencia, impulsando el empoderamiento de la población a través de metodologías participativas y utilizando herramientas de abordaje en el escenario comunitario desde la Atención Primaria.

4. Contribuir en el fortalecimiento de la Red iBIMEFA para la integración de investigadores de la Región, así como en la identificación y divulgación de oportunidades de formación y financiación y generar información y reuniones periódicas para protocolos concretos.

5. Mejorar canales de comunicación de las tesis/trabajos de grado/trabajos de campo que realiza estudiantes/residentes para conocimiento de la región en pro al seguimiento de los resultados como del conocimiento de este tipo de investigación y establecer repositorios de tesis/investigaciones y bases de datos para definición de líneas.

6. Incentivar/promover la Investigación clínica y epidemiológica con factor diferencial con fundamentos y principios de medicina familiar (uso de herramientas de Salud y Medicina Familiar) y retomar contacto con los subgrupos de cumbres anteriores, así coordinadores de grupos de la red IBIMEFA, identificando posibilidades concretas de financiación.

7. Enfatizar que es necesario que cada médico familiar tenga una población asignada específica, en un determinado territorio, y en todos los sectores del sistema de salud, que garantice el acceso y la equidad.

8. Recomendar que sea mínimo 30 médicos familiares por cada 100.000 habitantes, meta alcanzable en la siguiente decenio.

9. Recomendar que se diseñen estrategias comunes para los países miembros que permitan obtener indicadores estandarizados que evalúen el desempeño profesional y el impacto económico del médico familiar.

10. Gestionar alianzas estratégicas con los tomadores de decisiones, personal sanitario y la ciudadanía, con el fin de aplicar los principios de la prevención cuaternaria y disminuir el sobrediagnóstico y sobretratamiento, contribuyendo así al cuidado en salud con calidad.

11. Difundir en los equipos de salud y en la comunidad, a través de las redes sociales, medios de comunicación masiva, líderes comunitarios, escuelas y otros grupos de apoyo, el concepto de Prevención Cuaternaria, con el fin de lograr el "empoderamiento para la salud".

12. Incorporar y/o fortalecer, según sea el caso, la formación en salud mental y comunitaria necesaria para los espacios de atención en los que se desenvuelven los médicos de familia, sin patologizar las situaciones vitales, con el desarrollo de estrategias de autocuidado de las personas sustentables tanto en el tiempo como en la capacidad financiera de los países de Iberoamérica; con el fin de desarrollar la capacidad de enfrentar situaciones estresantes de la vida diaria, que permitan desarrollar fortalezas para situaciones de emergencias y desastres.

13. Fortalecer y empoderar el trabajo comunitario con el fin de que sea la propia comunidad empoderada quien establezca redes de apoyo en problemáticas de salud mental y esté preparada en conjunto para enfrentar situaciones de la vida diaria y le permita desarrollar acciones inmediatas en situaciones de emergencias y desastres naturales o no. 
14. Priorizar estrategias de autocuidado costo efectivas y orientadas a herramientas de desarrollo personal y de las familias, incluidos los equipos de salud, y los profesores, tutores de Medicina Familiar. De modo tal que se establezcan relaciones virtuosas orientadas a un trato cordial y constructivo. El cuerpo docente de las residencias de MF debe hacerse cargo de acciones tendientes al autocuidado de los alumnos, propendiendo a facilitar el proceso de aprendizaje y la preparación para ejercer la profesión en un medio tan complejo como son los centros de Atención Primaria de Salud o en cualquier contexto en el que se trabaje con la estrategia de APS.

15. Incluir el concepto de Prevención Cuaternaria en los programas académicos de pre grado y post grado, de las profesiones del campo de la salud, mediante la implementación de planes de estudio transversales, estimulando la investigación de la temática, acorde con las propuestas realizadas en las Cumbres y según las necesidades y regulaciones de cada país.

16. Reconocer el derecho a la salud de los migrantes y sus familias, asegurando el acceso igualitario, en las mismas condiciones de protección, amparo, y derechos que gozan los nacionales.

17. Incorporar en la malla curricular de los programas de pregrado, posgrado y formación continua, las competencias necesarias para la atención integral de la población migrante y de sus familias.

18. Crear un observatorio de Salud de la Migración, con registro de información confiable y validada, que permita análisis y seguimiento para la toma de decisiones efectivas y centradas en las personas y sus familias.
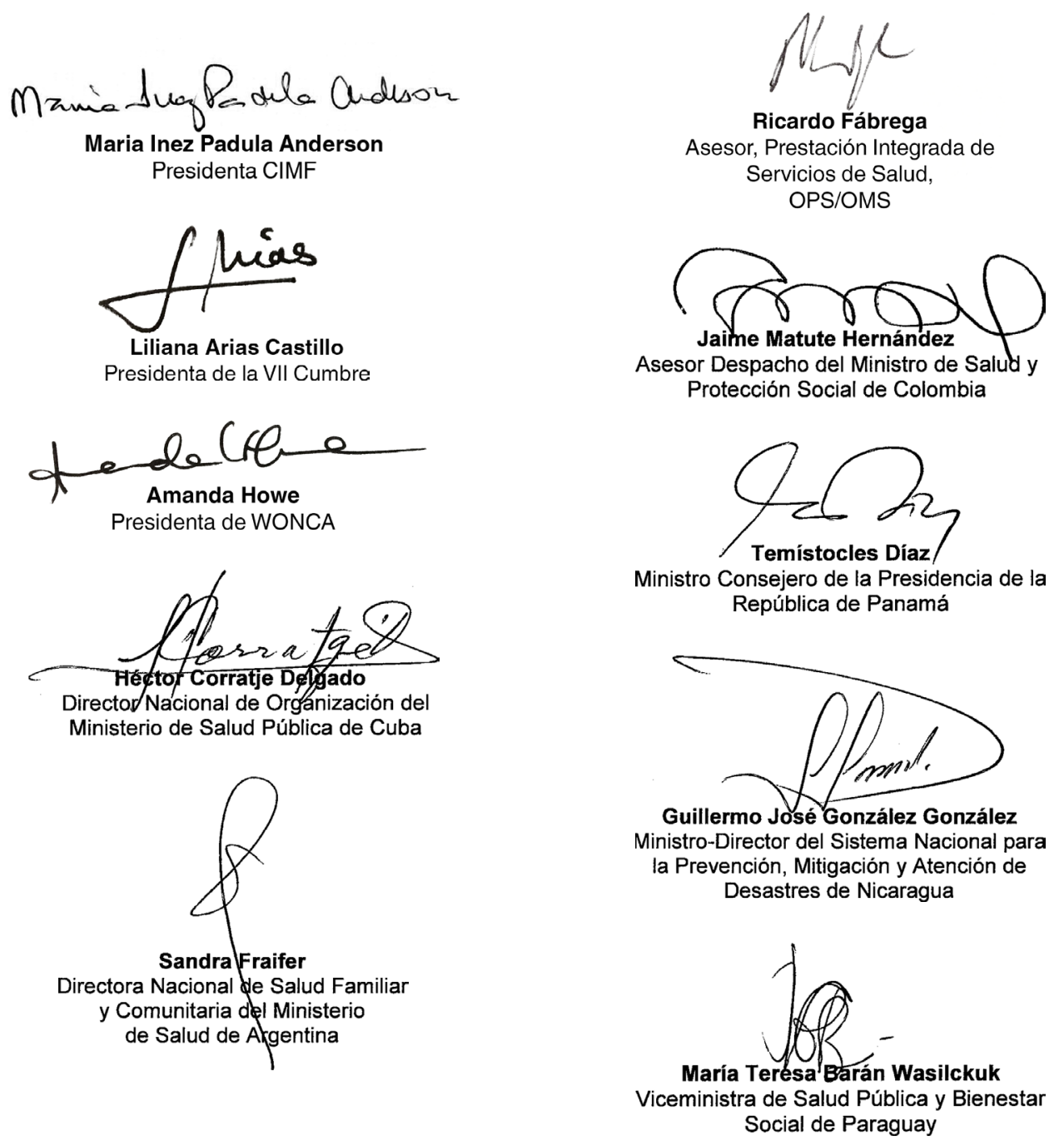